\title{
Inducing lucid dreams
}

During lucid dreams, sleeping individuals enter a state of consciousness in which they are aware that they are dreaming and can control dream events. Increased cortical activity in the low gamma frequency range $(\sim 40 \mathrm{~Hz})-$ particularly in frontal and temporal regions of the brain - has been observed during lucid dreaming. However, it is unknown whether this low gamma-band activity causes or results from lucid dreaming. Now, Voss et al. show that stimulation of sleepers' brains with $40 \mathrm{~Hz}$ currents during rapid eye movement (REM) sleep can induce lucid dreaming.

The authors applied frontotemporal transcranial alternating current stimulation (tACS) for 30 seconds at various frequencies to individuals in REM sleep. They found that individuals who underwent tACS at $25 \mathrm{~Hz}$ or $40 \mathrm{~Hz}$ - the lower gamma-band frequencies that were tested - exhibited a marked increase in electroencephalographic (EEG) activity at $\sim 25 \mathrm{~Hz}$ or $40 \mathrm{~Hz}$, respectively. Neither sham stimulation nor tACS at any of the other frequencies elicited any change in EEG activity.

Participants were awakened 5-10 seconds after tACS stimulation and asked to report and rate their dreams on scales of eight factors, three of which are known to be increased in lucid dreams. These include: insight (awareness of being in a dream); dissociation (experiencing the dream from a third-person perspective); and control of the dream's factors. Approximately one-third of $25 \mathrm{~Hz}$-stimulated individuals and about two-thirds of $40 \mathrm{~Hz}$-stimulated individuals experienced lucid dreams (defined by increased insight and dissociation scores), but no sham-stimulated individuals reported such dreams. These data show that lower gamma frequency tACS can induce lucid dreaming.

Interestingly, the increase in lower gamma frequency EEG activity that was induced by $25 \mathrm{~Hz}$ or $40 \mathrm{~Hz}$ stimulation was substantially higher in individuals who had experienced lucid dreams than in those who did not. Strikingly, the authors found that ratings of insight and dissociation strongly correlated with tACSinduced increases in $25 \mathrm{~Hz}$ and $40 \mathrm{~Hz}$ EEG activity. Together, these findings suggest that an increase in tACSinduced activity in the lower gamma frequency band is reciprocally associated with lucid dreaming.

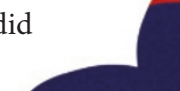

Overall, this study demonstrates that tACS during REM sleep can be used to induce low gamma-band EEG activity and lucid dreaming and that lower gamma-band activity is strongly associated with self-awareness.

Natasha Bray

ORIGINAL RESEARCH PAPER Voss, U. et al. Induction of self awareness in dreams through frontal low current stimulation of gamma activity. Nature Neurosci. http://dx.doi.org/10.1038/ nn. 3719 (2014)

\section{about two- \\ thirds of \\ $40 \mathrm{~Hz}-$ \\ stimulated \\ individuals \\ experienced \\ lucid dreams}

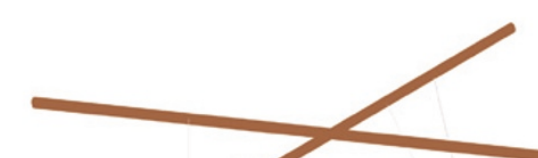

.

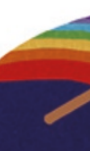

